ORIGINAL ARTICLE

\title{
Hospital based alternatives to acute paediatric admission: a systematic review
}

\section{Ogilvie}

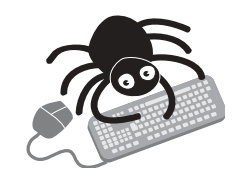

Supplementary material (including all tables) is available on the Archives of Disease in Childhood website (www. archdischild.com/ supplemental)

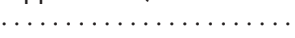

Correspondence to: Dr D Ogilvie, MRC Social \& Public Health Sciences Unit, University of Glasgow, 4 Lilybank Gardens, Glasgow G12 8RZ, UK; d.ogilvie@msoc. mrc.gla.ac.uk

Accepted 22 November 2003

\begin{abstract}
Aims: To synthesise published evidence of the impacts of introducing hospital based alternatives to acute paediatric admission.

Methods: Systematic review of studies of interventions for children with acute medical problems. Main outcome measures were: admission or discharge, unscheduled returns to hospital, satisfaction of parents and general practitioners, effects on health service activity, and costs.

Results: Twenty five studies were included: one randomised controlled trial, 23 observational or crosssectional studies, and one qualitative study. Many studies were of uncertain quality or were open to significant potential bias. About $40 \%$ of children attending acute assessment units in paediatric departments, and over $60 \%$ of those attending acute assessment units in A\&E departments, do not require inpatient admission. There is little evidence of serious clinical consequences in children discharged from these units, although up to $7 \%$ may subsequently return to hospital. There is some evidence that users are satisfied with these services and that they are associated with reductions in inpatient activity levels and certain hospital costs. Evidence about the impact of urgent outpatient clinics is very limited.

Conclusions: Current evidence supports a view that acute paediatric assessment services are a safe, efficient, and acceptable alternative to inpatient admission, but this evidence is of limited quantity and quality. Further research is required to confirm that this type of service reorganisation does not disadvantage children and their families, particularly where inpatient services are withdrawn from a hospital.
\end{abstract}

hildren's hospital admission rates have gradually risen in recent decades, but mean lengths of stay have fallen sharply. Most acute admissions now last for fewer than two days. These trends have led many paediatric departments to consider whether some episodes of acute illness could be safely managed without admitting the child to an inpatient ward at all, using alternative models of care (including services described as ambulatory care, intermediate care, or hospital at home) to provide a higher quality service for children. In some areas, ambulatory care services have also been introduced as a response to staffing difficulties in small or isolated inpatient units. ${ }^{1-5}$

In the UK, the Royal College of Paediatrics and Child Health (RCPCH) has identified two types of alternative service provided in hospitals:

- Acute assessment units (also known as emergency assessment, observation, or short stay units), which provide a rapid specialist consultation for acutely ill children in support of primary care services and A\&E departments, and provide in effect admission facilities for periods measured in hours rather than days

- Acute assessment clinics, which provide emergency outpatient consultations. ${ }^{3}$

Acute assessment units may be on the same hospital site as a paediatric inpatient unit, or on a different site (satellite units). The substitution of inpatient units with satellite units is often politically contentious. In the early 1990s it was proposed that children's day case services could replace small-tomedium-sized inpatient units. ${ }^{16}$ In 1996, a working party of the British Paediatric Association recommended that small units less than 30 minutes' journey from a larger inpatient unit should be closed and replaced by, for example, an emergency assessment unit, but acknowledged that this could impair access to services. ${ }^{2}$ More recent reports from the $\mathrm{RCPCH}$ have recognised that small inpatient units may have difficulty recruiting enough doctors to provide a safe service, but it has also been acknowledged that more research is needed on the acceptability and safety of alternatives to traditional inpatient services. ${ }^{3}$

Most existing evidence in this area relates to services for adults. $^{7}$ In this paper, I report the findings of a systematic review of studies of interventions to answer the question: what is the impact of introducing hospital-based alternatives to acute admission in medical paediatrics? I sought evidence of any effect on health or on health service process, including access to services, admissions and discharges, clinical outcomes, satisfaction or experiences of children, parents or health professionals, and economic effects.

\section{METHODS \\ Literature search}

I searched electronic databases, reference lists, and selected journals for reports in English of evaluation or audit studies of the impacts of any service provided in a hospital as an alternative to acute medical paediatric admission. Full details of the search strategy and inclusion criteria are available on the $A D C$ website.

\section{Quality assessment}

I first graded each article against the hierarchy of study designs specified by the NHS Centre for Reviews and Dissemination. ${ }^{8}$ No single quality assessment tool was suitable for use with all the studies. I therefore adapted a checklist from the CRD's lists for observational studies and 
applied this to all the studies, except for the one randomised controlled trial and one qualitative study, to which I applied the appropriate separate CRD checklists. ${ }^{8}$

\section{Synthesis}

The interventions, study designs, and impacts measured were diverse, and some studies did not report accurate numerical data. It was therefore inappropriate to attempt a formal statistical synthesis of the results. I summarised the findings using a series of tables and a narrative synthesis.

\section{RESULTS}

Detailed results tables are available on the $A D C$ website.

\section{Studies included}

Twenty five studies met the inclusion criteria, reported in 26 documents: 18 full papers from peer reviewed journals, five letters or commentaries in peer reviewed journals, and three other reports. ${ }^{35-32}$

The studies reported on interventions which fell into three main groups (table 1 ; see $A D C$ website):

- Acute assessment units based in a paediatric department (hereafter referred to as paediatric assessment units: 13 studies)

- Acute assessment units based in an accident and emergency department ( $A \& E$ assessment units: 9 studies)

- Acute assessment clinics (3 studies).

Six main types of investigation were reported (table 1). Apart from one randomised controlled trial and one qualitative study, all were quantitative observational studies of various types. Some studies included more than one approach.

Most studies concerned either children with acute medical problems or children attending A\&E departments. A few studies restricted their analysis to children with a single diagnosis (asthma) or a few common diagnoses. A few studies reported on units which also dealt with other cases such as medical or surgical day cases.

Some reports were very brief and contained scant details of methods. It was difficult to have confidence in attributing the impacts reported in many studies to the interventions described because the authors did not make comparisons between groups, did not adequately describe their methods, or did not address the possibility of biased comparisons.

Further details of the nature of the interventions and the quality assessment of the studies are summarised on the $A D C$ website.

\section{Patient outcomes}

Studies reporting patient outcomes generally involved the follow up of a complete cohort of patients attending a service over a period of time.

\section{Paediatric assessment units}

Discharge: In most studies, about $40 \%$ of children referred as emergencies were discharged without requiring inpatient admission (10 studies; table 3, $A D C$ website). This proportion was fairly consistent between studies except for that of Bothwell et al in Ulster, whose small study excluded the most unwell children, ${ }^{12}$ and the studies of two satellite units in England. ${ }^{5}{ }^{18}$ Doctors may have chosen not to refer very sick children to these units.

Unscheduled returns: Between $0.4 \%$ and $7 \%$ of discharged children returned unexpectedly to hospital (five studies: table 4, $A D C$ website), either with a worsening of the original problem or with an unrelated condition. The proportion returning increased with the length of study follow up.
Varying proportions of returning children then required admission. One study detailed 20 such admissions: 12 were for observation only, and four were for interventions lasting less than 24 hours. ${ }^{23}$

\section{A\&E assessment units}

Discharge: Most patients were discharged without requiring inpatient admission (eight studies: table 5, $A D C$ website). The proportion ranged from $62 \%$ to $99 \%$, largely appearing to reflect differences in case mix and definitions rather than an association with study quality. One study found that the proportion admitted was higher in a tertiary hospital $(6 \%)$ than in a general hospital $(4 \%) .{ }^{14}$

Inappropriate cases: Three studies reported that few (1-7\%) patients accepted for the acute assessment unit were subsequently considered inappropriate (meaning, for example, that patients spent over 24 hours in the unit, or required inpatient admission). ${ }^{1013} 14$ The two Australian studies identified no critical incidents within 72 hours of attending the units. ${ }^{13} 14$

Unscheduled returns: The same two studies found that $0.4 \%$ and $1.7 \%$, respectively, of patients discharged from the units returned for readmission within 72 hours. These were all described as having minor conditions. ${ }^{13}{ }^{14}$

Gouin et al compared the pattern of admission and reattendance before and after the introduction of a unit in Toronto. Children who attended the hospital with asthma were more likely to re-attend within 72 hours after the intervention $(5.0 \% v 3.2 \%)$, but re-attenders were less likely to be admitted $(28 \% \vee 39 \%) .^{20}$

In the randomised controlled trial by Willert et al, children with acute asthma were randomly assigned either to direct inpatient admission or to initial management in an acute assessment unit (holding room). Children discharged from the holding room were less likely to have a recurrence of asthma requiring further hospital treatment. ${ }^{32}$

\section{Acute assessment clinics}

The two brief reports of patient outcomes found that $13 \%$ and $19 \%$ of attenders, respectively, were admitted to hospital. ${ }^{9} 1617$ Another study, also reported briefly, found that $82 \%$ of referrals were subsequently deemed appropriate. ${ }^{27}$

\section{Changes in hospital admission patterns}

Studies reporting changes in hospital activity generally involved comparing activity in the year(s) before and after the new service was introduced. Most studies did not adjust for trends by making comparisons with a control hospital or population.

\section{Paediatric assessment units}

Several studies showed increasing demand on paediatric services during the study period, as measured by total annual numbers of referrals or admissions. This constituted an attempt to adjust for the confounding effect of secular trends.

Three studies (from two units) showed downward trends in the annual number of admissions for three years after the intervention..$^{22}{ }^{23} 26$ In Mid-Ulster, admissions from the local area fell by $47 \%$ between the pre-intervention year and the third post-intervention year. ${ }^{26}$

A further study found that the number and proportion of emergency admissions requiring an overnight stay decreased in the year after the intervention. ${ }^{11}$ Another found a decrease in the number of admissions, and in the proportion of children admitted from A\&E and/or the assessment unit in selected diagnostic groups, in the year after the intervention. $^{27}$ 


\section{A\&E assessment units}

One study showed that the rise in the paediatric admission rate stopped after the intervention, while the rate of A\&E attendances continued to rise. ${ }^{24}$

One study showed that a smaller proportion of children attending A\&E with asthma were admitted to hospital after the intervention $(24 \%)$ than before $(31 \%) .{ }^{20}$

The Australian studies showed that annual paediatric admissions and bed days fell by $10 \%$ and $15 \%$ respectively after the intervention. ${ }^{13} 14$

\section{Views and experiences of parents, GPs, and hospital staff}

These studies comprised five cross-sectional surveys and one in-depth qualitative study.

\section{Paediatric assessment units}

Graham et al found that parents in New Zealand were generally satisfied with their experiences. They detailed some specific problems which caused delays, including nursing and medical workload, dispensing of prescriptions, and patient transport inside the hospital..$^{21}$

Macleod et al found that parents and GPs in Mid-Ulster were generally satisfied with their experiences. All but one of the parents were satisfied or very satisfied with the unit and $82 \%$ felt that their child had benefited from not being admitted. Ninety seven per cent of the GPs were satisfied with the ease of access to the service and the promptness of the response. However, nearly half of the GPs did not agree that opening the unit had allayed their fears about the closure of the inpatient unit. ${ }^{26}$

Bothwell et al reported high levels of parental satisfaction in $95 \%$ of parents of attenders in Ulster, and noted that the dissatisfied parents were more likely to be in the group of parents whose children had been discharged rather than admitted. The proportion of parents who were satisfied remained high three weeks after discharge $(97 \%){ }^{12}$

Kibirige et al surveyed parents of children who were admitted from the acute assessment unit in Middlesbrough and found that $48 \%$ would have been happy to take their child home if greater home support had been available. Of those whose children were discharged from the unit, $82 \%$ were happy for a member of unit staff to make a follow up telephone call or visit. ${ }^{22}$

All the surveys of parents may have been subject to interviewer bias: the Mid-Ulster survey was conducted by the unit's nursing staff, and it was not clear who had carried out the interviews in the other surveys. The Mid-Ulster study may also have been biased by only surveying the parents of children who did not require admission: those whose children had to be transferred 22 miles to an inpatient unit may have had different views.

Turner's qualitative study found that parents were most satisfied when staff seemed approachable, listened, and discussed their child's care. Parents continued to need support after discharge. This included being given adequate information about their child's condition, knowing what to do if symptoms returned, and feeling able to telephone the unit for advice. ${ }^{30}$

\section{A\&E assessment units}

Leduc et al monitored patient complaints and surveyed nursing staff after introducing dedicated nursing staffing for the assessment unit in Denver. The incidence of complaints about the unit decreased by $50 \%$. All the nurses surveyed thought that families were more satisfied with the unit after the change. Most also thought that nurse-topatient ratios were safer $(96 \%)$ and that admissions to the unit were appropriate $(71 \%) .{ }^{25}$

\section{Economic impacts}

\section{Paediatric assessment units}

Four of the before-and-after studies of hospital activity included some assessment of economic impacts. One study showed a decrease in ward staffing costs and sickness absence following the opening of the unit, ${ }^{11}$ and two showed an associated decrease in bed days and by implication in inpatient costs. $^{13}{ }^{14}$ Kibirige et al showed that children discharged from the unit had fewer investigations than those who were admitted, suggesting that extra investigations were not being carried out in lieu of admitting those children.22

\section{A\&E assessment units}

The randomised controlled trial by Willert et al included a more comprehensive comparison of the direct and indirect costs of care for children managed in the acute assessment unit in Chicago with those for children admitted to the inpatient unit. They found no significant difference in indirect costs, but children who were managed initially in the acute assessment unit spent fewer days in hospital, had fewer days of intravenous therapy, and incurred lower room and therapy/ancillary charges. The comparison of room charges was biased because holding room charges were billed per hour, while inpatient charges were billed per whole day. The methods for estimating indirect costs were not stated. ${ }^{32}$

\section{Summary of key findings}

Many of the studies identified were of uncertain quality or were open to significant potential bias. The available evidence suggests that about $40 \%$ of children attending acute assessment units in paediatric departments, and over $60 \%$ of those attending acute assessment units in A\&E departments, do not require inpatient admission. There is little evidence of serious clinical consequences in children discharged from these units, although up to $7 \%$ may subsequently return to hospital. There is some evidence that users are satisfied with these services and that they are associated with reductions in inpatient activity levels and certain hospital costs. Evidence about the impact of urgent outpatient clinics is very limited.

\section{DISCUSSION}

This systematic review has aimed to synthesise evidence about the health impacts of one type of service reorganisation in acute paediatric care. Case studies of the processes and implications of organisational change in UK acute paediatric services have recently been reviewed in more detail elsewhere. $^{18}$

In order to make comparisons between the findings of evaluation studies and draw generalisable conclusions from them, it is necessary to understand clearly the context, as well as the content, of the "black box" of apparently successful interventions. ${ }^{33}$ There are several problems with the group of studies included in this review which limit the conclusions that can be drawn.

Some authors did not clearly describe key features of services which are highly likely to influence their performance, such as the criteria for acceptance, transfer, and discharge of patients, the clinical practice in their unit, or the means by which critical incidents are audited. Without such information, it is not possible to draw general conclusions about how these features affect outcomes for children. However, hospitals developing similar units can draw on Turner's qualitative findings in drawing up local clinical governance arrangements to assure the safety of children whose condition may deteriorate after initial assessment. ${ }^{30}$ This is particularly important in a specialty in which a high proportion of emergency cases present in the evenings or at 
night and in which outcomes cannot necessarily be reliably predicted from the presenting problem. ${ }^{34}$

Few studies had attempted to make an unbiased comparison of outcomes between services offered to similar groups of children. In particular, there were no studies comparing "traditional" inpatient care with a paediatric assessment unit operating at the same time. Children's use of emergency hospital services may be affected by sociospatial factors such as deprivation and proximity to hospital, and variables such as length of stay are dependent on the ages and case mix of children admitted. ${ }^{34-36}$ Factors such as these are likely to underlie at least some of the differences in patient outcomes between studies, but the reports generally contained insufficient information to assess their relative importance.

In the absence of robust comparative studies, external benchmarks drawn from routine activity data might be used for comparison, but this approach is not straightforward either. For example, the overall incidence of paediatric emergency readmission within seven days of discharge in England in 2001 was $5.5 \%$, but this performance indicator varied fivefold between the hospital trusts with the highest and lowest rates, and most of the studies in this review used different follow up periods. ${ }^{37}$

There is little consensus about the meaning of terms used or about the collection of routine hospital activity data on acute assessment episodes. For example, the meaning of the term "admission" varies between countries; ${ }^{38}$ in the studies in this review, paediatric assessment units were typically "admitting" patients for two to four hours whereas the A\&E units were more likely to hold children for up to 24 hours. Even within the UK, there is still a need for clearly defined activity measures, and the potential pitfalls of misinterpreting routine paediatric inpatient statistics have been highlighted..$^{18} 36$

Although some studies included a retrospective assessment of the appropriateness of admissions, other work has shown that measures of appropriateness are context dependent and of only modest reliability. ${ }^{38}{ }^{39}$ It is also generally assumed that children discharged from an acute assessment unit would have been admitted if the unit had not been there, although it is also possible that the opening of the unit might have altered the threshold for referral. No study has adequately investigated this.

Most intervention research in this field has taken a relatively limited perspective on what outcomes should be evaluated.$^{40}$ I found few studies that thoroughly examined possible adverse outcomes, the perspective of service users, or economic impacts. Furthermore, many of the potential impacts of this type of service reorganisation on the population remain unknown. In particular, we lack evidence of how replacing inpatient units with satellite units affects the population's access to health care, how users' views about acute assessment services compare with those about traditional inpatient care, and whether clinical outcomes in general are affected by introducing new services.

Future studies should aim to address these gaps in the evidence. These are likely to require more rigorous methods, which may include the experimental or quasi-experimental comparison of outcomes for cohorts of children exposed to different types of acute paediatric service; sampling the experiences of parents and children in larger surveys less prone to interviewer and selection bias; and adopting a stronger population perspective on evaluation.

\section{Conclusion}

Current evidence supports a view that acute paediatric assessment services are a safe, efficient, and acceptable alternative to inpatient admission, but this evidence is of limited quantity and quality. Further research is required to confirm that this type of service reorganisation does not disadvantage children and their families, particularly where inpatient services are withdrawn from a hospital.

\section{ACKNOWLEDGEMENTS}

This article is based on work originally carried out for Lanarkshire NHS Board and which formed part of a successful submission for the examination for Membership of the Faculty of Public Health Medicine. I thank Charles Clark, Mark Petticrew, Jim Miller, Oliver Blatchford, and my examiners and referees for their comments on this work at various stages in its development.

Competing interests: none declared

\section{REFERENCES}

1 British Paediatric Association. Flexible options for paediatric care: a discussion document. London: British Paediatric Association, 1993.

2 British Paediatric Association. Future configuration of paediatric services: report of a working party. London: British Paediatric Association, 1996.

3 Royal College of Paediatrics \& Child Health. Ambulatory paediatric services in the UK: report of a working party. London: Royal College of Paediatrics \& Child Health, 1998.

4 Royal College of Paediatrics \& Child Health. Looking ahead: paediatrics and child health-the next ten years. London: Royal College of Paediatrics \& Child Health, 2001

5 Royal College of Paediatrics \& Child Health. Old problems, new solutions: 21 st century children's healthcare. London: Royal College of Paediatrics \& Child Health, 2002.

6 Taylor B. How many inpatient paediatric units do we need? Arch Dis Child 1994:71:360-4.

7 Hider P. Acute medical admissions: a critical appraisal of the literature. Christchurch: New Zealand Health Technology Assessment, 1998.

8 NHS CRD. Undertaking systematic reviews of research on effectiveness. Report no. 4, 2nd edn. York: NHS Centre for Reviews and Dissemination, 2001.

9 Baildam E, Ewing C. Ambulatory paediatrics-making a difference [commentary]. Arch Dis Child 1997;76:468-76.

10 Beattie T, Moir P. Paediatric accident \& emergency short-stay ward: a 1-year audit. Arch Emerg Med 1993;10:181-6.

11 Beverley D, Ball R, Smith R, et al. Planning for the future: the experience of implementing a children's day assessment unit in a district general hospital. Arch Dis Child 1997;77:287-92.

12 Bothwell J, Bell A, Sheridan P. Ambulatory paediatrics: improving patient care [letter]. Irish Med J 2001;94:186.

13 Browne G, Penna A. Short stay facilities: the future of efficient paediatric emergency services. Arch Dis Child 1996;74:309-13

14 Browne G. A short stay or 23-hour ward in a general and academic children's hospital: are they effective? Pediatr Emerg Care 2000;16:223-9.

15 Carter E. Ambulatory paediatrics-making a difference [commentary]. Arch Dis Child 1997;76:468-76.

16 Coleman H, Finlay F. Emergency consultation clinics avert unnecessary admissions [letter]. BMJ 1996;312:1102.

17 Coleman H, Finlay F. The rapid access paediatric clinic: a way to reduce inappropriate admissions to hospital. Prof Care Mother Child 1997:7:157-9.

18 Cresswell T. A review of recently developed models for acute child health services with ambulatory components. Stockton-on-Tees: Northern \& Yorkshire Public Health Observatory, 2002.

19 Dawson K, Mogridge N, Abbott G. A paediatric day unit: the first year's experience. N Z Med J 1991;104:185-7.

20 Gouin S, Macarthur C, Parkin P, et al. Effect of a pediatric observation unit on the rate of hospitalization for asthma. Ann Emerg Med 1997;29:218-22.

21 Graham D, Abbott G, Dawson K. A paediatric day ward: parental expectations, perceptions, and satisfaction. N Z Med J 1991;104:405-7.

22 Kibirige S, Edmond K, Kibirige J, et al. A seven year experience of medical emergencies in the assessment unit. Arch Dis Child 2003:88:125-9.

23 Lal $M$, Kibirige $M$. Unscheduled return visits within 72 hours to an assessment unit. Arch Dis Child 1999;80:455-8.

24 Lamireau T, Llanas B, Fayon M. A short stay observation unit improves care in the paediatric emergency care setting [letter]. Arch Dis Child 2000;83:369.

25 Leduc K, Haley-Andrews S, Rannie M. An observation unit in a pediatric emergency department: one children's hospital's experience. J Emerg Nurs 2002;28:407-13

26 Macleod C, Jenkins J, Kennedy F, et al. Ambulatory paediatrics: does it work? Irish Med J 2002;95:41-4

27 Meates M. Ambulatory paediatrics-making a difference. Arch Dis Child 1997;76:468-76

28 Scribano P, Wiley J, Platt K. Use of an observation unit by a pediatric emergency department for common pediatric illnesses. Pediatr Emerg Care $2001 ; 17: 321-3$.

29 Smith R, Eastham E, Stokoe L, et al. A paediatric medical day unit- 10 years experience. Matern Child Health 1993:143-7. 
30 Turner G. Parents' experiences of ambulatory care. Paediatr Nurs 1998;10:12-13,16.

31 Wiley J, Friday J, Nowakowski T, et al. Observation units: the role of an outpatient extended treatment site in pediatric care. Pediatr Emerg Care 1998;14:444-7.

32 Willert C, Davis A, Herman J, et al. Short-term holding room treatment of asthmatic children. J Pediatr 1985; 106:707.

33 Rychetnik L, Frommer $M$, Hawe $P$, et al. Criteria for evaluating evidence on public health interventions. J Epidemiol Community Health 2002;56:119-27.

34 Stewart M, Werneke U, MacFaul R, et al. Medical and social factors associated with the admission and discharge of acutely ill children. Arch Dis Child 1998;79:219-24.

35 Beattie T, Gorman D, Walker J. The association between deprivation levels, attendance rate and triage category of children attending a children's accident and emergency department. Emerg Med J 2001;18:110-11.

36 MacFaul R, Werneke U. Recent trends in hospital use by children in England. Arch Dis Child $2001: 85: 203-7$.

37 Department of Health. NHS performance ratings and indicators: July 2002, http://www.doh.gov.uk/performanceratings/2002//accessed 25 September 2003).

38 Werneke U, Smith H, Smith I, et al. Validation of the paediatric appropriateness evaluation protocol in British practice. Arch Dis Child 1997;77:294-8.

39 Werneke U, MacFaul R. Evaluation of appropriateness of paediatric admission. Arch Dis Child 1996;74:268-73.

$40 \varnothing$ øretveit J. Evaluating health interventions. Buckingham: Open University Press, 1998.

\section{ARCHIVIST}

\section{Retractile testes: follow up study}

T

he prevalence of retractile testes in school age boys has been estimated at between 4 and 13 per 1000 boys. Such testes have completed the descent process and can be brought down into the scrotum by manipulation but, when released, retract into the inguinal canal by cremasteric contraction. Although retractile testes are commonly regarded as normal there is still debate about their management. It is not known, for instance, how long each testis spends in the inguinal canal and whether the increase in surrounding temperature could be harmful. The experience of one paediatric surgeon has been reported (Journal of Pediatric Surgery 2004;39:1014-7).

The series included 150 consecutive boys referred between April 1982 and April 1999 with a primary care diagnosis of retractile testis or cryptorchidism. The average age at referral was 5.2 years and average follow up 3.8 years. There were 205 retractile testes; 58 on the right, 37 on the left, and 55 bilateral cases. Six boys had a contralateral undescended testis and 32 had an inguinal hernia. Orchidopexy was performed if the testis became fixed in a high position or if it failed to grow satisfactorily: it was performed in 37 boys, in 34 because the testis could no longer be brought into the scrotum and in three because of failure of testicular growth. Greater difficulty in manipulating the testis into the scrotum was a predictor of eventual orchidopexy. Orchidopexy was performed in $69 \%$ of boys with an associated hernia and $9 \%$ without. One patient who had cleft palate and duplication of the ureter on the same side as the retractile testis had normal descent of the testis but at the age of 16 developed testicular cancer in the previously retractile testis.

In this series of boys referred to a surgeon 37 of 150 boys came to orchidopexy. The authors of the paper conclude that all boys with retractile testes should be followed up (although 93 of the 205 testes were normally placed in the scrotum and only three of those came to orchidopexy). They suggest, oddly, that the follow up might be done on the boys' birthdays. "Happy surgical appointment day, young man." 